\title{
FAUNA EDÁFICA DE ÁREA DEGRADADA REVEGETADA COM PINHÃO MANSO EM MONOCULTIVO E CONSÓRCIO COM Andropogon gayanos L.
}

\author{
Milton Marques Fernandes ${ }^{1}$, Marcos Emanuel Costa Veloso ${ }^{2}$, Marcelo Duarte Silva ${ }^{3}$, Márcia \\ Rodrigues de Moura Fernandes ${ }^{4}$ \& Nara Núbia Cruz Lima ${ }^{5}$
}

RESUMO: Este trabalho objetivou avaliar os efeitos comparativos da revegetação com pinhão-manso em monocultivo ou consorciado com a gramínea Andropogon gayanos L. em relação a uma área desertificada (testemunha) sobre a fauna edáfica de uma região localizada no Sul do Piauí. Foram instaladas armadilhas tipo pitt fall para a coleta da fauna do solo no período chuvoso e no período seco. A área de consórcio de pinhão manso com Andropogon apresentou maior atividade e número de grupos taxonômicos da fauna edáfica em comparação com o plantio em monocultivo e a área desertificada durante período seco. A área de plantio de pinhão-manso em monocultivo não apresentou diferença na diversidade de Shannon da fauna edáfica quando comparado à área desertificada.

PALAVRAS-CHAVE: organismos do solo, desertificação, Jatropha curcas L.

\section{EDAPHIC WILD LIFE IN AREAS OF Jatrophacurcas L. MONOCULTURE AND ITS CONSORTIUM WITH Andropogon gayanos L.}

ABSTRACT: This study aimed to evaluate the comparative effect son the soil fauna of an area revegetated with jatropha monoculture,with an consortium of jatropha and Andropogon gayanos L., and a desertified area(control), all located in southern Piaui. We installed Pittfal traps to collect soil fauna in the rainy and dry seasons. Theare a with the consortium of jatropha and Andropogon had a higher activity and higher number of soil fauna taxa compared to the monoculture and desertified areas, during the dry season. The area of Jatropha monoculture showed no difference in Shannon diversity of soil fauna when compared to the desertified area.

KEYWORDS: soil organisms, desertification, Jatropha curcas L.

\section{INTRODUÇÃO}

Cerca de $1.340 .000 \mathrm{~km}^{2}$ do território brasileiro é composto de áreas suscetíveis à desertificação, incluindo a região semiárida, subúmido seco e áreas de entorno. No Piauí, $1.241 \mathrm{~km}^{2}$ do território se encontra desertificado. O município de Gilbués, no sudoeste do Estado, é um dos que mais sofrem com este processo. (SILVA, 2012).

Segundo Sales (2003), o processo de desertificação na região de Gilbués, iniciou-se com a exploração desordenada do garimpo de diamante, na década de 40,

\footnotetext{
${ }^{1}$ Universidade Federal de Sergipe. E-mail: miltonmf@gmail.com

${ }^{2}$ EMBRAPA MEIO NORTE. E-mail: mcars@embrapa.br

${ }^{3}$ Engenheiro Agronomo, Doutor em irrigação e Hidrulica, Pesquisador da EMBRAPA MEIO NORTE-TEresina-PI. E-mail: mduarte@bol.com.br

${ }^{4}$ Engenheiro Agronomo pela Universidade Estadual do Piauí, Mestre em Solos e Nutrição de Plantas pela UFPI. E-mail: marcia86@bol.com.br

${ }^{5}$ Engenheira Florestal pela UFPI, Mestranda em Ciências Florestais pela Universidade de Brasilia. E-mail: naranubia25@bol.com.br
}

acompanhada de outros fatores, dentre esses se destacam: gêneses do solo, superpastoreio, uso do fogo desordenado, desmatamento generalizado, agricultura inadequada e as altas taxas de precipitações pluviais no período chuvoso.

Uma das soluções para combate a desertificação são as gramíneas como o Andropogon gayanos L., pois apresentam rápido crescimento, baixa exigência em fertilidade e o sistema radicular que proporciona melhor suporte mecânico e adiciona grande quantidade de matéria orgânica (PEREIRA, 2006).

O pinhão-manso (Jatropha curcas L.) é uma espécie arbórea perene e monóica, pertencente à família das Euphorbiaceae. É uma planta tolerante a seca (LAVIOLA e DIAS, 2008; SATURNINO et al., 2005; DIAS et al., 2007), com ampla adaptação edafoclimática e capaz de se desenvolver e produzir mesmo em terrenos marginais. 
Dentre os ambientes em que o pinhão manso pode se desenvolver, estão os solos arenosos, pedregosos, salinos, alcalinos e rochosos os quais, sob o ponto de vista nutricional e físico são restritivos ao pleno desenvolvimento de raízes. Tais características credenciam o seu plantio visando o controle de erosão, a contenção de encostas e dunas ao longo de canais, rodovias, ferrovias e como cerca viva em divisões internas ou limites de propriedades rurais (SATURNINO et al., 2005). É grande o seu potencial na reabilitação de ecossistemas naturais degradados e/ou desertificados (FRIGO et al., 2009).

Os ecossistemas naturais comumente seguem o princípio de que a maior diversidade de espécies de organismos, plantas e animais permite uma maior resistência à perturbação e à interferência. Os ecossistemas com alta diversidade tendem a se recuperar mais rapidamente da perturbação e restaurar o equilíbrio em seus processos de ciclagem de nutrientes e fluxo de energia. Já nos ecossistemas com mais baixa diversidade, a perturbação pode provocar mais facilmente modificações permanentes no funcionamento resultando na perda de recursos do ecossistema e em alterações na constituição de espécies (ALTEMBURG et al., 2010).

A abundância e diversidade de espécies da fauna edáfica são indicadores de homeostase do ecossistema (PEREIRA et al., 2012). O aumento do número de indivíduos de espécies da fauna ocorre pela disponibilidade de melhores condições ambientais, que favorecem a reprodução dos invertebrados (SEEBER et al., 2005). Este fato pode ser verificado por meio do índice de diversidade de Shannon e do índice de uniformidade como Pielou (BROWN et al., 2004).

A fauna edáfica apresenta uma complexidade nos diferentes grupos taxonômicos e funcionais diretamente relacionados ao tipo de ambiente. Por sua sensibilidade às modificações que ocorrem no ambiente a fauna pode ser considerada como indicador das condições encontradas no solo (DORAN e ZEISS, 2000; FERNANDES et al., 2011).

Neste sentido, este trabalho objetivou avaliar os efeitos comparativos da revegetação com pinhão-manso em monocultivo ou consorciado com a gramínea Andropogon gayanos L. em relação a uma área desertificada (testemunha) sobre a fauna edáfica de uma região localizada no Sul do Piauí.

\section{MATERIAL E MÉTODOS}

O experimento foi implantando em 2009 em uma área experimental da EMBRAPA Meio-Norte localizado no Município de Gilbués - PI e o estudo avaliado no ano de 2011, sob as coordenadas geográficas 945’55', latitude Sul e 4521'00'” longitude Oeste. O solo das áreas de estudo é do tipo Neossolo Litólico eutrófico (SALES, 1998).

Segundo Vieira et al. (2007) o clima, segundo a classificação de Köppen, é do tipo Aw, megatérmico, com moderada deficiência hídrica no inverno. A temperatura oscila entre a mínima de $25{ }^{\circ} \mathrm{C}$ e máxima de $35{ }^{\circ} \mathrm{C}$, sendo que o mês mais frio apresenta média superior a $18{ }^{\circ} \mathrm{C}$. A precipitação média anual varia de 800 a $1200 \mathrm{~mm}$. O período chuvoso se estende de outubro a maio e os meses mais úmidos são de novembro a março (VIEIRA et al., 2007).

Para o estudo foram selecionadas três coberturas: (1) plantio de pinhão manso plantada em novembro de 2009 em uma área em processo de desertificação, (2) pinhão manso consorciado com gramínea da espécie Andropogon (Andropogon gayanus) plantada em novembro de 2009 em uma área em processo de desertificação e (3) uma área de Cerrado (testemunha) em processo de desertificação com a presença de árvores esparsas e a presença de ravinas e pequenas voçorocas localizadas ao lado dos plantios sem cobertura. O plantio do pinhão-manso foi realizado em filas simples, utilizando um espaçamento de 4,0 x 3,0 m, com uma população de plantas de 1667 plantas por hectare, em ambas as áreas.

Em cada área foram instaladas armadilhas do tipo pitt fall utilizadas para a avaliação da atividade da fauna epígea (MOLDENKE, 1994). Na confecção dos pitt fall foi utilizado um pote com 0,11 m de diâmetro e 0,075 m de profundidade.

Também se utilizou pratos plásticos com 0,15 m de diâmetro para cobrir os potes e proteger contra a ação da chuva. No interior de cada pote foi adicionada uma solução de formol a $4 \%$ para preservação da fauna no período de permanência das armadilhas. As mesmas foram instaladas na interface serrapilheira-solo e permaneceram durante sete dias no campo em uma área de 1 hectare (ha). Decorrido este tempo fez-se a triagem com auxílio de lupa e chaves taxonômicas, com quantificação do número total de indivíduos, grupos funcionais, atividade da fauna epígea (indivíduos/armadilhas/dia).

A fauna epígea foi avaliada pelo número de indivíduos por armadilha por dia, riqueza da fauna, que corresponde ao número de grupos identificados, e riqueza media que representa o número médio de indivíduos por armadilha. O número total de grupos taxonômicos presentes foi avaliado pelo índice de diversidade de Shannon que foi calculado por meio da seguinte formula: $\mathrm{H}=-\Sigma p i{ }_{\mathrm{x}} \log _{2}$ pi, em que pi e a proporção de indivíduos que pertence a "iésima" família. Para a análise da uniformidade, ou seja, a abundância relativa utilizou-se o índice de Uniformidade de Pielou, por meio da seguinte expressão: $\mathrm{U}=\mathrm{H} / \operatorname{lognS}$, onde $\mathrm{H}$ corresponde ao índice de Shannon, e o número total de indivíduos na comunidade e S é o numero total de espécies encontradas em cada área estudada.

Os organismos também foram divididos em grupos funcionais, conforme propostos por Costa (2002), a partir de características ao uso do habitat e à principal forma de utilização do recurso alimentar, sendo estes: 
Macrófagos, Formicidae, Holometabólicos; Saprófagos, Isoptera, Predadores, Parasitóides e Fitófagos.

As áreas foram revegetadas em novembro de 2009, e após dois anos da implantação dos plantios nas áreas foram realizadas as coletas da fauna do solo realizadas no período chuvoso (Fevereiro) e no período seco em Setembro de 2011. Os dados de números de indivíduos, grupos taxonômicos, atividade da fauna epígea e os índices de diversidade de Shannon, Pielou e Riqueza de grupos foram submetidos a análise de variância para verificar a normalidade dos dados e a comparação de médias, entre cada área, foi feita por meio da aplicação do teste Tukey a $5 \%$ pelo software Sisvar (FERREIRA, 2000).

\section{RESULTADOS E DISCUSSÃO}

Na área desertificada, durante o período chuvoso, foram observados 33 indivíduos, pertencentes a nove grupos taxonômicos. Os grupos taxonômicos que mais se destacaram foram os Formicidae (30,3\%), Orthoptera $(24,2 \%)$ e Diptera $(18,2 \%)$ que somados, equivalem a $72,7 \%$ das comunidades estudadas. No plantio de pinhão-manso em monocultivo (ou solteiro), o número de indivíduos coletados foi de 112, pertencentes a oito grupos taxonômicos. Os grupos taxonômicos de maior destaque foram os Formicidae (34,2\%), Orthoptera (21,4\%), Coleoptera (16,9\%) e Diptera (13,4\%), correspondendo a $85,9 \%$ do total. Já no plantio de pinhão-manso consorciado com Andropogon gayanus, observa-se que o número de indivíduos foi de 170, pertencentes a sete grupos taxonômicos no período chuvoso. Os grupos taxonômicos de maior destaque foram os Formicidae (42,8\%), Coleoptera (22,9\%) e Diptera $(14,7 \%)$ e Orthoptera $(8,2 \%)$, correspondendo a 88,6\% do total (Tabela 1).

Durante o período seco, na área desertificada, foram observados 51 indivíduos, pertencentes a quatro grupos taxonômicos. Os grupos taxonômicos de maior destaque foram Formicidae (80,1\%) e os Coleoptera (5,9\%), que somados equivalem a $86,0 \%$ das comunidades estudadas na área. No mesmo período, em plantio de pinhão-manso foram observados 52 indivíduos, pertencentes a quatro grupos taxonômicos. O grupo taxonômico de maior destaque foi Formicidae correspondendo a 92,0 \% das comunidades estudadas. Já no plantio pinhão-manso consorciado com Andropogon gayanus foram observados 318 indivíduos, pertencentes a cinco grupos. O grupo taxonômico de maior destaque foi Formicidae respondendo por 93,8\% das comunidades estudadas na área (Tabela 1).
Tabela 1 - Distribuição relativa (\%) dos grupos taxonômicos da fauna edáfica na área desertificada $(A D)$, área de plantio de pinhão manso em monocultivo (Pi) e pinhão-manso consorciado com Andropogon gayanus (Pi-Ca), durante os períodos chuvoso e seco.

\begin{tabular}{llll}
\hline \multirow{2}{*}{ Grupos taxonômicos } & AD & Pi & Pi-Ca \\
\cline { 2 - 4 } & \multicolumn{3}{c}{ Chuvoso } \\
\hline Araneae & 6,1 & 0,0 & 0,6 \\
Coleoptera & 3,0 & 16,9 & 22,9 \\
Dermaptera & 3,0 & 0,0 & 0,0 \\
Diplopoda & 6,1 & 5,5 & 0,0 \\
Diptera & 18,2 & 13,4 & 14,8 \\
Formicidae & 30,3 & 34,2 & 42,8 \\
Hemyptera & 6,1 & 3,5 & 5,3 \\
Hymenoptera & 3,0 & 3,3 & 1,3 \\
Oligochaeta & 0,0 & 1,8 & 4,1 \\
Orthoptera & 24,2 & 21,4 & 8,2 \\
\hline \multirow{2}{*}{ Grupos taxonômicos } & $\mathrm{AD}$ & $\mathrm{Pi}$ & $\mathrm{Pi}-\mathrm{Ca}$ \\
\cline { 2 - 4 } & \multicolumn{3}{c}{$\mathrm{Seco}$} \\
\hline Araneae & 0,0 & 0,0 & 0,9 \\
Coleoptera & 5,9 & 1,9 & 3,1 \\
Dermaptera & 2,0 & 0,0 & 0,0 \\
Diplopoda & 0,0 & 0,0 & 0,0 \\
Diptera & 0,0 & 1,9 & 0,3 \\
Formicidae & 80,1 & 92,0 & 93,8 \\
Hemyptera & 0,0 & 0,0 & 0,6 \\
Hymenoptera & 8,1 & 2,3 & 1,3 \\
Miriapoda & 0,0 & 0,0 & 0,0 \\
Oligochaeta & 0,0 & 0,0 & 0,0 \\
Orthoptera & 3,9 & 1,9 & 0,0 \\
\hline
\end{tabular}

Os resultados demonstraram que Formicidae foi o grupo taxonômico mais numeroso em ambos os períodos avaliados (seco e chuvoso). Porém, embora os percentuais de indivíduos do grupo por período tenham se mantido próximas, o número de indivíduos variou sensivelmente entre os períodos seco e chuvoso. Em ambos os períodos estudados, o número de indivíduos observados nas áreas de consórcio de pinhão-manso com Andropogon gayanus e de pinhão-manso em monocultivo foi superior ao observado na área desertificada.

Nunes et al. (2008), avaliando a recolonização da fauna edáfica em áreas de caatinga submetidas a queimadas cobertas com diferentes vegetais e tempo de repouso, observaram que os grupos Formicidae e Coleoptera foram mais abundantes em todos os sistemas de manejo e que as áreas mais perturbadas apresentaram menor número de indivíduos, principalmente no período seco. Fernandes et al. (2011) avaliando a influência de diferentes coberturas florestais observou que Formicidae foi predominante na área mais preservada que em plantios de espécies florestais para recuperação de áreas degradadas. Observa-se neste estudo comportamento semelhante, visto que as áreas mais preservadas (consórcio de pinhão-manso com Andropogon gayanus e pinhão-manso em monocultivo) apresentaram maior 
número de indivíduos do grupo Formicidae que a área mais desertificada (Tabela 1).

Tabela 2 - Porcentagem de indivíduos coletados na área desertificada $(A D)$ e nas áreas de plantio de pinhão manso (Pi), pinhão manso consorciado com capim Andropogon (Pi-Ca) para os diferentes grupos funcionais.

\begin{tabular}{cccc}
\hline \multirow{2}{*}{ Grupos Funcionais } & $\mathrm{AD}$ & $\mathrm{Pi}$ & $\mathrm{Pi}-\mathrm{Ca}$ \\
\cline { 2 - 4 } & \multicolumn{3}{c}{ Chuvoso } \\
\cline { 2 - 4 } Sociais: Formicidae & 47,8 & 51,2 & 48,4 \\
Holometabólicos & 30,4 & 41,5 & 40,6 \\
Saprófagos & 8,8 & 2,4 & 4,5 \\
Predadores & 13,0 & 4,9 & 6,5 \\
\hline \multirow{2}{*}{ Grupos Funcionais } & $\mathrm{AD}$ & $\mathrm{Pi}$ & $\mathrm{Pi}-\mathrm{Ca}$ \\
\cline { 2 - 4 } & \multicolumn{3}{c}{$\mathrm{Seco}$} \\
Sociais: Formicidae & 88,2 & 94,2 & 95,0 \\
Holometabólicos & 5,9 & 3,9 & 3,5 \\
Predadores & 2,0 & 0,0 & 0,9 \\
Fitófagos & 3,9 & 1,9 & 0,6 \\
\hline
\end{tabular}

Os grupos funcionais sociais Formicidae e Holometabólicos foram dominantes entre os indivíduos coletados no período chuvoso, respondendo por mais de $70,0 \%$ do total efetivo das comunidades estudadas nas três áreas em estudo (Tabela 2). Observa-se que, durante o período chuvoso, o grupo funcional Holometabólicos apresentou maior percentual nas áreas de plantio de pinhão-manso em monocultivo e consórcio pinhãomanso com Andropogon gayanus que a área desertificada (Tabela 2). Calvi et al. (2010) observaram maior percentual do grupo funcional Holometabólicos em florestas secundárias no período chuvoso demonstrando que o solo e o ecossistema possuem um habitat bem estruturado.

Os grupos funcionais sociais Formicidae e Holometabólicos foram dominantes entre os indivíduos coletados no período seco, respondendo por mais de $90,0 \%$ do total efetivo das comunidades estudadas, nas três áreas em estudo. Observa-se que o grupo funcional social Formicidae foi maior nas áreas de plantio de pinhão-manso em monocultivo e pinhão-manso consórcio com Andropogon gayanus, que na área desertificada (Tabela 2). De acordo com Pasqualin et al., (2012), a manutenção de uma camada de serapilheira durante período seco nas áreas de plantio de pinhãomanso em monocultivo e pinhão-manso em consórcio com Andropogon gayanus favorecem maior percentual do grupo funcional social Formicidae em detrimento da área desertificada.

Observa-se que a área desertificada obteve maiores índices de diversidade de Shannon, Pielou e riqueza de grupos taxonômicos diferindo significativamente no período chuvoso que as demais áreas estudadas (pinhãomanso e pinhão manso consorciado com Andropogon
(Tabela 3). É provável que nas áreas de pinhão-manso e pinhão-manso consorciado com Andropogon exista o predomínio de poucos grupos taxonômicos com maior número de indivíduos que afeta negativamente os índices de Shannon, Pielou e riqueza de grupos taxonômicos. Candido et al. (2012) observou que uma área em recuperação apresentou menores índices de Shannon, Pielou e riqueza em relação a mata ciliar devido a dominância de alguns grupos taxonômicos na área em recuperação (Tabela 3).

Em relação aos índices de Shannon, Pielou e riqueza de grupos no período seco, verificou-se um equilíbrio entre as áreas de plantio com pinhão-manso, pinhão-manso consorciado com Andropogon e a área desertificada não apresentando diferença estatística significativa. Desta forma, observa-se que a revegetação com pinhão-manso em monocultivo e o seu consorciamento com capim Andropogon não foi capaz de manter maior umidade que a área desertificada no período de maior estresse hídrico influenciando negativamente nos índices de Shannon, Pielou e a riqueza de grupos da fauna do solo (Tabela 3). Souza et al. (2008), estudando diferentes coberturas vegetais em uma restinga no Rio de Janeiro, observaram que a umidade do solo é mais determinante na estrutura da comunidade da fauna do solo que a diferença de cobertura vegetal quando o teor de umidade do solo é baixo. Observa-se que a baixa diversidade de espécies utilizadas na revegetação nas áreas de plantio de pinhãomanso e pinhão consorciado com Andropogon resulta em similaridade com a área desertificada (Tabela 3). Quanto maior a complexidade da vegetação maior o número de espécies da fauna do solo devido a maior disponibilidade de micro-hábitats (VICENTE et al., 2010).

A área de pinhão-manso consorciado com Andropogon apresentou maior atividade no período chuvoso e no seco que as demais áreas apresentando diferenças estatísticas. A área de plantio de pinhão-manso foi semelhante à área desertificada no período seco e diferiu significativamente no período chuvoso. Dessa forma, observa-se que o consórcio com Andropogon no plantio de pinhão manso influenciou positivamente na atividade da fauna do solo no período chuvoso (Tabela 3). A presença de gramíneas mantém um considerável aporte orgânico resultante da renovação e aporte de serapilheira. Esse material funciona como fonte de alimento e na maioria dos casos cria um microclima favorável aos invertebrados do solo assemelhando-se a um ambiente natural de floresta aumentando a atividade da fauna do solo (NUNES et al., 2012). 
Tabela 3 - Índices ecológicos de Shannon, Pielou, Riqueza e Atividade (indivíduos/ armadilhas/dia) da fauna do solo na área desertificada, plantio de pinhão manso e plantio de pinhão manso consorciado com capim Andropogon.

\begin{tabular}{cccc}
\hline Índices Ecológicos & AD & Pi & Pi-Ca \\
\hline & \multicolumn{3}{c}{ Chuvoso } \\
\hline Shannon & $2,41 \mathrm{a}$ & $1,25 \mathrm{~b}$ & $1,17 \mathrm{~b}$ \\
Pielou & $2,72 \mathrm{a}$ & $1,49 \mathrm{~b}$ & $0,47 \mathrm{~b}$ \\
Riqueza & $4,69 \mathrm{a}$ & $2,94 \mathrm{~b}$ & $2,69 \mathrm{~b}$ \\
Atividade & $0,94 \mathrm{c}$ & $3,20 \mathrm{~b}$ & $4,85 \mathrm{a}$ \\
\hline & \multicolumn{3}{c}{ Seco } \\
\hline Shannon & $0,20 \mathrm{a}$ & $0,12 \mathrm{a}$ & $0,19 \mathrm{a}$ \\
Pielou & $0,34 \mathrm{a}$ & $0,20 \mathrm{a}$ & $0,27 \mathrm{a}$ \\
Riqueza & $1,75 \mathrm{a}$ & $1,74 \mathrm{a}$ & $1,59 \mathrm{a}$ \\
Atividade & $1,45 \mathrm{~b}$ & $1,48 \mathrm{~b}$ & $9,08 \mathrm{a}$ \\
\hline
\end{tabular}

De acordo com a análise de agrupamento observou-se que a área pinhão-manso consorciado com Andropogon no período seco diferiu das demais áreas demonstrando que o uso de uma gramínea em consorcio com o pinhãomanso fornece melhores condições para a fauna epígea no período seco. Corrêa e Bento (2010), observaram que a revegetação de uma área minerada com quatro tipos de gramíneas proporcionaram melhorias nos atributos da fauna do solo.

A área desertificada e a área de plantio de pinhão-manso apresentaram grande similaridade em torno de $97 \%$ no período seco (Figura 1). No período chuvoso a área desertificada apresentou boa similaridade com a área de plantio de pinhão-manso de $85 \%$ de similaridade. Ainda no período chuvoso observa-se que a área pinhão-manso consorciado com Andropogon forma um agrupamento diferenciado com as demais áreas, mas ainda assim apresenta uma similaridade de 75\% (Figura1).

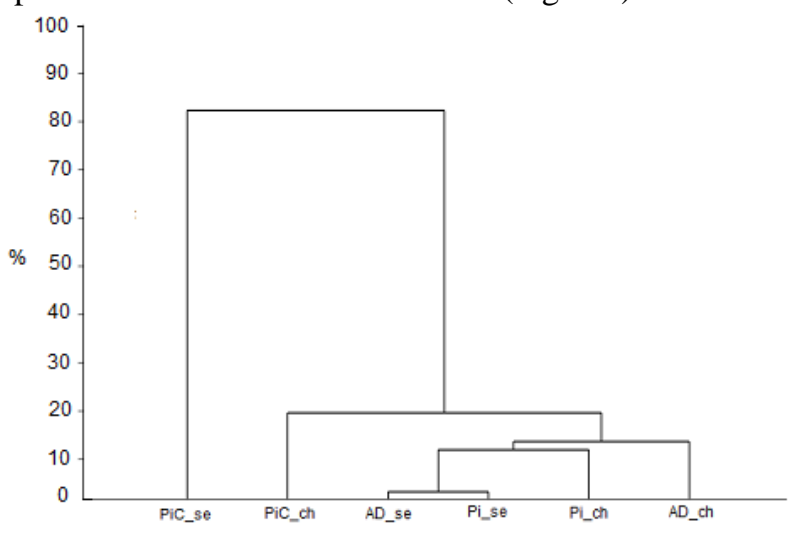

Figura 1 - Dendrograma apresentando a distância entre a área desertificada no período seco (AD_se) e chuvoso ( $A D \_c h$ ), plantio de pinhão manso consorciado com Andropogon no período seco (PiC_se) e chuvoso (PiC_ch) e áreas de plantio de pinhão manso no período seco (Pi_se) e chuvoso (Pi_ch) a partir da análise das comunidades da fauna baseado em distância euclidiana.

\section{CONCLUSÕES}

A área de consórcio de Andropogon com plantio de pinhão-manso apresentou maior atividade e número de grupos taxonômicos da fauna edáfica em comparação com o plantio pinhão-manso em monocultivo e a área desertificada durante período seco.

A área de plantio de pinhão-manso em monocultivo não apresentou diferença na diversidade de Shannon da fauna edáfica quando comparado à área desertificada.

\section{REFERÊNCIAS}

ALTEMBURG, S. G. N.; LUZZARDI, R. E. S.; LOVATTO, P. O. Importante papel da biodiversidade biológica na qualidade do solo em sistemas de produção agrícola: um enfoque para a microbiologia edáfica como biondicadora. Caderno de Pesquisa, Santa Cruz do Sul, v. 2, n. 2, p. 18-36, 2010. (Série Biologia).

BROWN, G. G.; MORENO, A. G.; BAROIS, I.; FRAGOSO, C.; ROJAS, P.; HERNÁNDEZ, B.; PATRÓN, J. C. Soil macrofauna in SE Mexican pastures and the effect of conversion from native to introduced pastures. Agriculture, Ecosystems and Environment, Amsterdã, v. 103, n.2, p. 313-327, 2004.

CALVI, G. P.; PEREIRA, M. G.; ESPINDULA JUNIOR, A.; MACHADO, D. L. Composição da fauna edáfica em duas áreas de floresta em Santa Maria de Jetibá - ES, Brasil. Acta Agronomica, Bogotá, v. 59, n. 59, p. 37-45, 2010.

CANDIDO, A. K. A.; SILVA N. M.; BARBOSA, D. S.; FARIAS, L. N.; SOUZA, W. P. Fauna edáfica como bioindicadores de qualidade ambiental na nascente do rio São Lourenço, Campo Verde -MT, Brasil. Engenharia Ambiental, Espírito Santo do Pinhal, v. 9, n. 1, p. 67-82, 2012.

COSTA, P. Fauna do solo em plantios experimentais de Eucalyptus grandis Maiden, Pseudosamanea guachapele Dugand e Acacia mangium Willd. 2002. 93 f. Dissertação (Mestrado em Ciência do Solo)Universidade Federal Rural do Rio de Janeiro. Seropédica, 2002.

CORRÊA, R. S.; BENTO, M. A. B. Qualidade do substrato minerado de uma área de empréstimo revegetada no Distrito Federal. Revista Brasileira de Ciência do Solo, Viçosa, MG, v. 34, n.4, p. 1435-1443, 2010.

DIAS, L. A. S.; LEME, L. P.; LAVIOLA, B. G.; PALLINI FILHO, A.; PEREIRA, O. L.; CARVALHO, M.; MANFIO, C. E.; SANTOS, A. S.; SOUSA, L. C. A.; OLIVEIRA, T. S.; DIAS, D. C. F. S. Cultivo de Pinhão Manso (Jatropha curcas L.) para produção de óleo combustível. Viçosa: UFV, 2007. v. 1, 40 p. 
DORAN, J. W.; ZEISS, M. R. Soil Health and sustainability: managing the biotic component of soil quality. Applied Soil Ecology, Lincoln, v. 15, p. 3-11, 2000 .

FERNANDES, M. M.; MAGALHÃES, L. M. S.; PEREIRA, M. G.; CORREIA, M. E. F.; BRITO, R. J.; MOURA, M. R. Influência de diferentes coberturas florestais na fauna do solo na FLONA Mário Xavier, no município de Seropédica, RJ. Revista Floresta, Curitiba, v. 41, n. 3, p. 533-540, 2011.

FERREIRA, D. F.Sistemas de análise estatística para dados balanceados. Lavras: UFLA/DEX/SISVAR, 2000. 145 p.

FRIGO, M. S.;BUENO, O. C.;ESPERANCINI, M. S. T.;FRIGO, E. P. A cultura do pinhão-manso (Jatropha curcas L. curcas L.): uso para fins combustíveis e descrição agronômica. Varia Scientia, Cascavel, v. 7, p. 47-63, 2009.

LAVIOLA, B. G.; DIAS, L. A. Teor e acúmulo de nutrientes em folhas e frutos de pinhão manso. Revista Brasileira de Ciência do Solo, Viçosa, MG, v. 32, p. 1969-1975, 2008.

MOLDENKE, A. R. Arthropods. In: WEAVER, R. W.; ANGLE, S.; BOTTOMLEY, P.; BEZDICEK, D.; SMITH, S.; TABATABAI, A.; WOLLUM, A. (Ed.). Methods of soil analysis: microbial and biochemical properties. Madison: SSSA, 1994. Part 2, p. 517-542.

NUNES, L. A. P. L.; SILVA, D. I. B.; ARAUJO, A. S. F.; LEITE, L. F. C.; CORREIA, M. E. F. Caracterização da fauna edáfica em sistemas de manejo para produção de forragens no Estado do Piauí. Revista Ciência Agronômica, Fortaleza, v.43, n.1, p. 30-37, 2012.

NUNES, L. A. P. L.; ARAUJO FILHO, J. A.; MENEZES, R. I. Q. Recolonização da fauna edáfica em áreas de caatinga submetida a queimadas. Revista Caatinga, Mossoró, v. 21, n.3, p. 214-220, 2008.

PASQUALIN, L. A.; DIONÍSIO, J. A.; ZAWADNEAK, M. A. C.; MARÇAL, C. T. Macrofauna edáfica em lavouras de cana-de-açúcar e mata no noroeste do Paraná - Brasil, Semina: Ciências Agrárias, Londrina, v. 33, n. 1, p. 7-18, 2012.

PEREIRA, A. R. Como selecionar plantas para áreas degradadas e controle de erosão. Belo Horizonte: FAPI, 2006. 70 p.

PEREIRA, M. G.;SILVA, A. N.; PAULA, R. R.; MENEZES, L. F. T. Aporte e decomposição do folhedo em floresta periodicamente inundável na Restinga da Marambaia, RJ. Ciência Florestal, Santa Maria, v. 22, n.1, p. 59-67, 2012.
SALES, M. C. L. Degradação ambiental em Gilbués-PI. Mercator: Revista de Geografia da UFC, Fortaleza, v. 2, n. 4, p. 115-124, 2003.

SALES, M. C. L. Estudo da degradação ambiental em Gilbués-PI: reavaliando o "Núcleo de desertificação". 1998. 86 f. Dissertação (Mestrado em Geografia)Universidade de São Paulo, São Paulo, 1998.

SATURNINO, H. M.; PACHECO, D. D.; KAKIDA, J.; TOMINAGA, N.; GONÇALVES, N. P. Cultura do pinhão-manso (Jatropha curcas L. curcas L.). Informe Agropecuário: EPAMIG, Belo Horizonte, v. 26, n. 229, p. 44-73, 2005.

SEEBER, J.; SEEBER, G. U. H.; KÖSSLER, W.; LANGEL, R.; SCHEU, S.; MEYER, E. Abundance and trophic structure of macrodecomposers on alpine pastureland (Central Alps, Tyrol): effects of abandonment of pasturing. Pedobiologia, New York, v. 49, p. 221-228, 2005.

SILVA, M. D. Atributos químicos e biológicos de um Neossolo Litólico Eutrófico cultivado com pinhão manso e gramíneas, em área degradada no município de Gilbués, PI. 2012. 63 f. Dissertação (Mestrado em Agronomia/Solos e Nutrição de Plantas)-Universidade Federal do Piauí, Bom Jesus, 2012.

SOUZA, R. C.; CORREIA, M. E. F.; PEREIRA, M. G.; SILVA, E. M. R.; PAULA, R. R.; MENEZES, L. F. T. Estrutura da comunidade da fauna edáfica em fragmentos florestais na Restinga da Marambaia, RJ. Revista Brasileira de Ciências Agrárias, Recife, v. 3, p. 30-39, 2008.

VICENTE, N. M. F.; CURTINHAS, J. N.; PEREZ, A. L.; PREZOTTI, L. Fauna edáfica auxiliando a recuperação de áreas degradadas do Córrego Brejaúba, MG. Revista Floresta e Ambiente, Seropédica, v. 2, n. 17, p.104-110, 2010.

VIEIRA, V. C. B.; SALVIANO, A. A. C.; COSTA, E. F.; SILVA, F. B.; CORDEIRO, E.; MELO, L. F. S.; FERREIRA, G. B. T. F. Mapeamento de áreas degradadas na região do cerrado do Sul do Piaú́. Teresina: Convênio Fundação Agente/CODEVASF, 2007. 75 p. (Relatório Técnico) 